\title{
Research on Nonlinear Dynamics of Drive Mode in Z-Axis Silicon Microgyroscope
}

\author{
Lu Xu, ${ }^{1,2}$ Hongsheng Li, ${ }^{1,2}$ Jia Liu,, ${ }^{1,2}$ Yunfang Ni, ${ }^{1,2}$ and Libin Huang \\ ${ }^{1}$ School of Instrument Science and Engineering, Southeast University, Nanjing 210096, China \\ ${ }^{2}$ Key Laboratory of Micro-Inertial Instrument and Advanced Navigation Technology of Ministry of Education, \\ Southeast University, Nanjing 210096, China
}

Correspondence should be addressed to Hongsheng Li; hsli@seu.edu.cn

Received 15 May 2014; Accepted 21 July 2014; Published 11 August 2014

Academic Editor: Ignacio R. Matias

Copyright (C) $2014 \mathrm{Lu} \mathrm{Xu}$ et al. This is an open access article distributed under the Creative Commons Attribution License, which permits unrestricted use, distribution, and reproduction in any medium, provided the original work is properly cited.

\begin{abstract}
A novel drive method is proposed to increase the vibration amplitude of drive mode of silicon vibratory gyroscopes based on the nonlinear dynamics research made in this paper. A nonlinear dynamic equation, considering the nonlinear mechanical-elastic and electrostatic force, was firstly deduced according to the simplified structure schematic of drive mode. Then, experiments were made to characterize the nonlinear amplitude-frequency response characteristic as well as its dependence on drive voltage. Besides, by solving the nondimensional nonlinear dynamics using Matlab and Simulink, the influence of initial status of drive mode, that is, the displacement and the velocity, on the stable vibration amplitude was presented. The amplitude-frequency characteristic was also analyzed by simulation. The result of simulation shows good agreement with that of experiments. When nonlinearity is present in the amplitude-frequency characteristic, there is a wide flat region with larger amplitude than the peak amplitude obtained in the linear characteristic. Finally, a novel drive method was presented to excite the nonlinear vibration of large amplitude in the downwards-sweeping characteristic curve. It is numerically proved that this merit can be utilized to enlarge the drive mode's vibration amplitude so as to enhance silicon gyroscopes' performance.
\end{abstract}

\section{Introduction}

As part of a new generation of inertial sensors, silicon microgyroscopes, used for measurement of angular rate or angle of a rotating carrier, have a lot of merits, such as low power consumption, small volume and weight, high shock resistivity, and suitability for batch fabrication [15]. However, the performance of silicon microgyroscopes is lower than conventional mechanical, rotating wheel, fiberoptical, and ring laser gyroscopes [6]. Thus, it is necessary to improve the performance so as to broaden their application market.

Large vibration amplitude of drive mode is one of the conditions for silicon gyroscopes to achieve high performance $[7,8]$. Therefore, capacitive actuators of more combs and smaller gaps are designed in microgyroscopes so as to increase the vibration amplitude when the same drive voltage is applied. However, when gaps become small and the vibration amplitude gets large, the nonlinear terms of the force applied on proof mass can become sufficiently high to bend the amplitude-frequency characteristic curve. For the traditional drive methodology, drive mode works at the natural frequency in the linear amplitude-frequency characteristic curve. However, when the amplitude-frequency characteristic curve is nonlinear, the drive methodology for the drive mode of silicon gyroscopes is not yet noticed.

Nonlinear dynamics of MEMS resonators has been studied analytically, numerically, and experimentally by some institutions [9-14]. In these literatures, dynamic models are constructed to match the theoretical characteristic curves with the experimental phenomena of real resonator samples $[11,12]$. The models can be solved by different methods, such as the theoretical method of multiple scales [10], the semianalytical harmonic method [9], the numerical integration method [9-11], and the electrical equivalent-circuit modelling method [14]. It is known that the nonlinear 


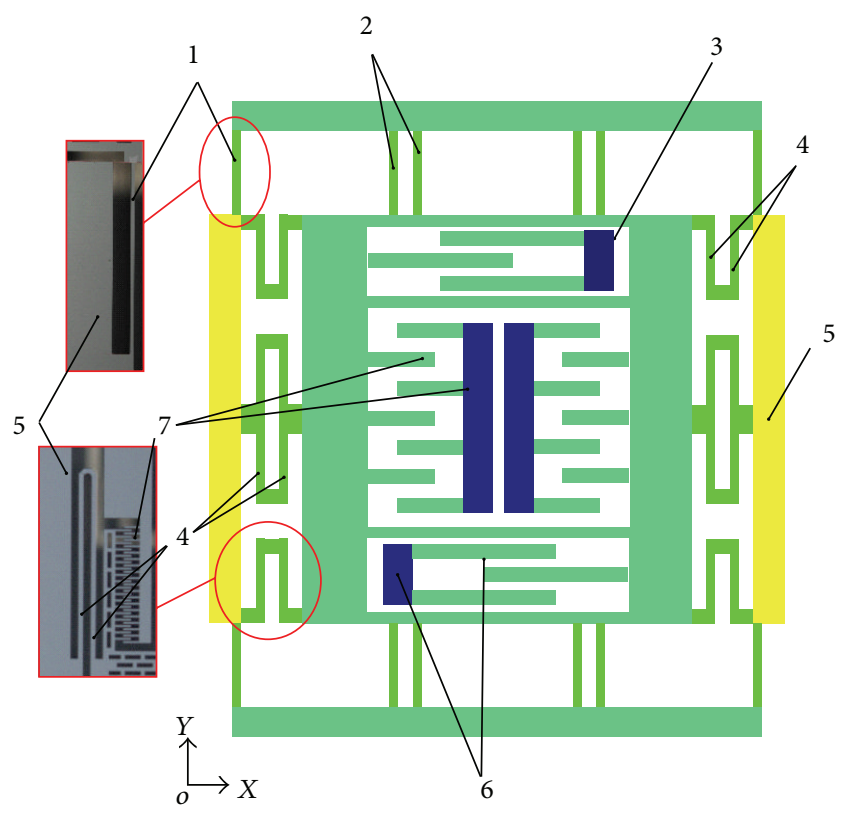

Figure 1: Simplified schematic of drive mode in $Z$-axis silicon gyroscope. 1. Beam 1: straight beam, 2. Beam 2: parallel combination, 3. proof mass, 4. Beam 3: serial combination, 5. anchor, 6. sense comb, and 7. drive comb.

phenomena refer to the spring softening and hardening phenomena in the amplitude-frequency curves. In capacitive MEMS resonators, the phenomena mainly result from the nonlinear mechanical-elastic reaction force when the mechanical support system is out of its linear work range, as well as the nonlinear electrostatic actuation force produced when the squeeze gaps of capacitors are small. As the drive mode of gyroscope is considered as a resonator, some more research is made in this paper on the nonlinear characteristics of the gyroscope prototype designed by our research team. Based on the nonlinear amplitude-frequency characteristic, a novel drive method is provided to make drive mode vibrate at large amplitude.

The outline of the paper is as follows. First, in Section 2, the nonlinear dynamics is derived based on the simplified schematic of the drive mode. Then, in Section 3, the experimental amplitude-frequency curves are obtained by using a detect circuit and sweeping the actuation frequency increasingly and decreasingly, separately. Besides, the nonlinear phenomena are also illustrated by solving the dynamics numerically using the Matlab and Simulink model in Section 4. Finally, in Section 5, a novel drive method is proposed for actuating the vibration of large amplitude in the nonlinear amplitude-frequency characteristic curve.

\section{Dynamics of Drive Mode}

2.1. Structure Schematic for Drive Mode. In the $Z$-axis silicon microgyroscope designed by our research team, the proof mass is supported by straight beams, as well as U-shaped beams, and driven and detected by capacitors of comb fingers
[15-17]. The dimensions of the gyroscope sample, fabricated by SOG process, are around $8 \mathrm{~mm} \times 8 \mathrm{~mm} \times 60 \mu \mathrm{m}$ and the SEM photos of beams and capacitors are displayed in Figure 1. Figure 1 shows the simplified structure schematic for the drive mode of the microgyroscope, consisting of the proof mass, comb actuators, sense combs, and supporting beams. As seen in the graph, Beam 1 is a fixed-guided straight beam, and Beam 2 and Beam 3 are the parallel and the serial combination of Beam 1, respectively. The proof mass is displaced by the electrostatic force produced when differential voltage is applied on drive electrodes. Since system's stiffness in the sense direction ( $Y$-axis) is much larger than that in the drive direction ( $X$-axis), the motion of drive mode is simplified as 1-DOF translation in the $X$-axis, as displayed in (1). $x$ is the displacement of the proof mass along the drive direction, $F_{e}$ and $F_{m}$ are the electrostatic force and the mechanical-elastic reaction force, and $F_{d}=c_{x} \dot{x}$ is the damping force with $c_{x}$ being the damping coefficient:

$$
m_{x} \ddot{x}=F_{e}-F_{m}-F_{d} .
$$

2.2. Mechanical-Elastic Reaction Force. In Figure 1, as the displacement of the proof mass in the drive direction is $x$, the deformation of the straight beam in Beams 3 is $x / 2$. Since the equivalent stiffness of Beam 2 is twice the stiffness of Beam 1 , the assumption is made that the deformation in Beam 1 is twice that in Beam 2. Thus, the deformation of the straight beam in Beams 1 and 2 is $2 x / 3$ and $x / 3$, respectively. The elastic reaction force applied on proof mass by all Beams 2 is approximated through a cubic polynomial relation, as displayed in (2) [10], where $E$ is the elastic modulus of silicon and $w, h$, and $l$ are the width, depth, and length of a straight beam. Similarly, the reaction force produced by all Beams 3 is shown in (3). Thus, the total mechanical-elastic reaction force applied on the proof mass in the drive direction is deduced in (4), where $K_{\text {linear }}$ is defined as the linear mechanical stiffness and $K_{\text {non-linear }}$ as the quadratic mechanical stiffness coefficient. Consider

$$
\begin{aligned}
F_{B 2} & =\frac{8}{3} \frac{E w^{3} h}{l^{3}} x+\frac{8}{27} \frac{E w^{3} h}{l^{2}} x^{3}, \\
F_{B 3} & =4 \frac{E w^{3} h}{l^{3}} x+\frac{E w^{3} h}{l^{2}} x^{3}, \\
F_{m} & =F_{B 2}+F_{B 3} \\
& =\frac{20}{3} \frac{E w^{3} h}{l^{3}} x+\frac{35}{27} \frac{E w^{3} h}{l^{2}} x^{3} \\
& =\left(K_{\text {linear }}+K_{\text {non-linear }} x^{2}\right) x .
\end{aligned}
$$

2.3. Electrostatic Force. Two drive electrodes in Figure 1 are applied with differential voltages $V_{a} \sin 2 \pi f_{d} t+V_{d}$ and $V_{a} \sin 2 \pi f_{d} t-V_{d}$ relative to the proof mass, in which $V_{d}$ is the DC bias, $V_{a}$ is the amplitude of AC voltage, and $f_{d}$ is the actuation frequency. $N$ groups of comb fingers are attached to one drive stator. Figure 2 illustrates one group of comb fingers, made up of one moving comb and two fixed combs. As explained in Section 2.1, the moving combs are assumed to 


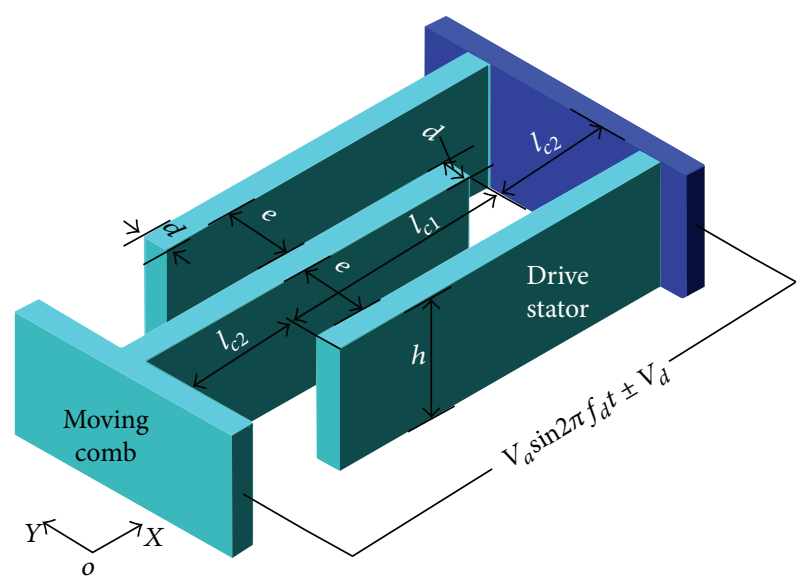

Figure 2: One group of comb fingers.

vibrate only in the $x$-axis. Then, the electrostatic force applied on the proof mass along the $x$-axis is derived in (5) [5]. $F_{h}$ is generated by the overlapped areas with a slide gap of $e$ and $F_{y}$ is generated by overlapped areas with a squeeze gap of $l_{c 2}$. It is easily noticed that $F_{h}$ is independent of $x$ while $F_{y}$ has a nonlinear relationship with it. Thus, the nonlinear electrostatic force applied on proof mass is relevant to the squeeze gap $l_{c 2}$.

2.4. Nonlinear Dynamics. Substituting (4) and (5) into (1), the nonlinear dynamics for drive mode is displayed in (6), which can be simplified as (7) when $V_{d}$ is much larger than $V_{a}$. Based on (7), the natural frequency of drive mode is approximated in (8), where $K_{\text {elinear }}=6 \mathrm{~N} \varepsilon d h V_{d}^{2} / l_{c 2}^{2}$ is the linear electroelastic stiffness and $K_{2 \text { enon-linear }}=12 N \varepsilon d h V_{d}^{2} / l_{c 2}^{5}$ is the quadratic electroelastic stiffness coefficient. It is easily seen that the natural frequency increases with the mechanical-elastic stiffness and decreases with the electroelastic stiffness. Besides, the mechanical-elastic and electroelastic stiffness both cause the nonlinearity between the natural frequency and the displacement. The nondimensional differential dynamics, as seen in (9), is obtained from (6) by the replacement of $\bar{x}=$ $x / l_{c 2}, \bar{t}=\widetilde{\omega}_{x} t=2 \pi \tilde{f}_{x} t, \tilde{f}_{x}=(1 / 2 \pi) \sqrt{K_{\text {linear }} / m_{x}}$, and $Q_{x}=2 \pi \tilde{f}_{x} \cdot m_{x} / c_{x}$. The nondimensional dynamics is used in the following numeric analysis:

$$
\begin{gathered}
F_{e}=\underbrace{\frac{4 V_{d} V_{a} N \varepsilon h}{e} \sin 2 \pi f_{d} t}_{F_{h}}+\underbrace{\frac{3 N \varepsilon d h}{2}\left[\frac{\left(V_{d}+V_{a} \sin 2 \pi f_{d} t\right)^{2}}{\left(l_{c 2}-x\right)^{2}}-\frac{\left(V_{d}-V_{a} \sin 2 \pi f_{d} t\right)^{2}}{\left(l_{c 2}+x\right)^{2}}\right]}_{F_{y}}, \\
m_{x} \ddot{x}+c_{x} \dot{x}+K_{\text {linear }} x+K_{\text {non-linear }} x^{3} \\
=\frac{4 V_{d} V_{a} N \varepsilon h}{e} \sin 2 \pi f_{d} t+\frac{3 N \varepsilon d h}{2}\left[\frac{\left(V_{d}+V_{a} \sin 2 \pi f_{d} t\right)^{2}}{\left(l_{c 2}-x\right)^{2}}-\frac{\left(V_{d}-V_{a} \sin 2 \pi f_{d} t\right)^{2}}{\left(l_{c 2}+x\right)^{2}}\right], \\
m_{x} \ddot{x}+c_{x} \dot{x}+K_{\text {linear }} x+K_{\text {non-linear }} x^{3}=\frac{4 V_{d} V_{a} N \varepsilon h}{e} \sin 2 \pi f_{d} t+\frac{3 N \varepsilon d h V_{d}^{2}}{2}\left[\frac{1}{\left(l_{c 2}-x\right)^{2}}-\frac{1}{\left(l_{c 2}+x\right)^{2}}\right], \\
\bar{x}^{\prime \prime}+\frac{1}{Q_{x}} \bar{x}^{\prime}+\bar{x}+\frac{l_{c 2}^{2} K_{\text {non-linear }} \bar{x}^{3}}{m_{x} \widetilde{\omega}_{x}^{2}} \\
=\frac{4 V_{d} V_{a} N \varepsilon h}{e l_{c 2} m_{x} \widetilde{\omega}_{x}^{2}} \sin \frac{f_{d}}{\widetilde{f}_{x}} \bar{t}+\frac{3 N \varepsilon d h}{2 l_{c 2}^{3} m_{x} \widetilde{\omega}_{x}^{2}}\left[\frac{\left(V_{d}+V_{a} \sin \left(f_{d} / \tilde{f}_{x}\right) \bar{t}\right)^{2}}{(1-\bar{x})^{2}}-\frac{\left(V_{d}-V_{a} \sin \left(f_{d} / \tilde{f}_{x}\right) \bar{t}\right)^{2}}{(1+\bar{x})^{2}}\right] .
\end{gathered}
$$

\section{Experimental Characterization}

3.1. Experimental Setup. As presented in Figure 3, the carrier voltage $V_{c}=V_{a} \sin 2 \pi f_{d} t$ is applied on the anchor, that is, the moving combs, and two differential sense electrodes are connected with their own preamplifiers. The current, $i_{s 1}$ and $i_{s 2}$, is integrated and the output voltage, $V_{+}$and $V_{-}$, passes through the differential amplifier. The process is shown as below:

$$
\begin{aligned}
V_{o}=V_{+}-V_{-} & =\frac{1}{C_{f}} \int i_{s 1} d t-\frac{1}{C_{f}} \int i_{s 2} d t \\
& =\frac{1}{C_{f}} \int\left(\frac{d q_{s 1}}{d t}-\frac{d q_{s 2}}{d t}\right) d t
\end{aligned}
$$




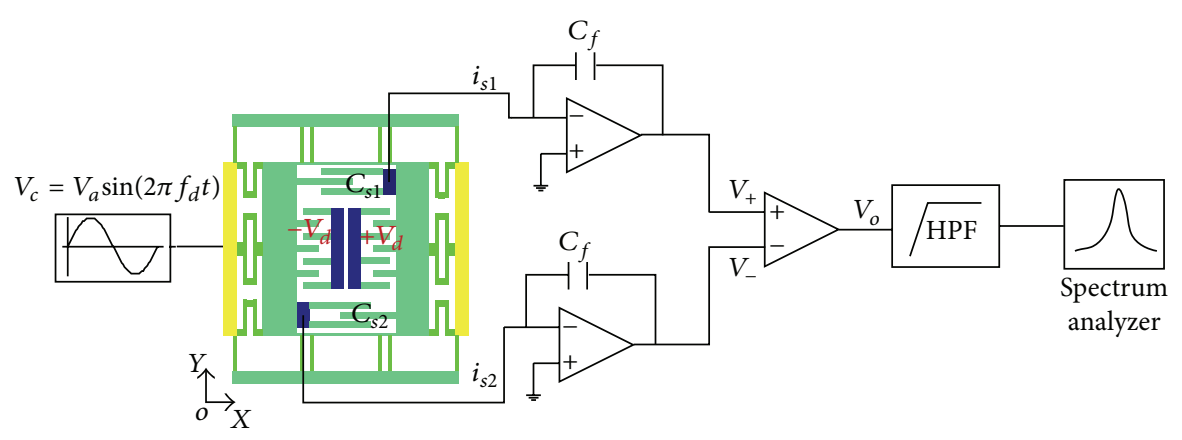

FIGURE 3: Schematic of signal-detecting interface circuitry for drive mode vibration.

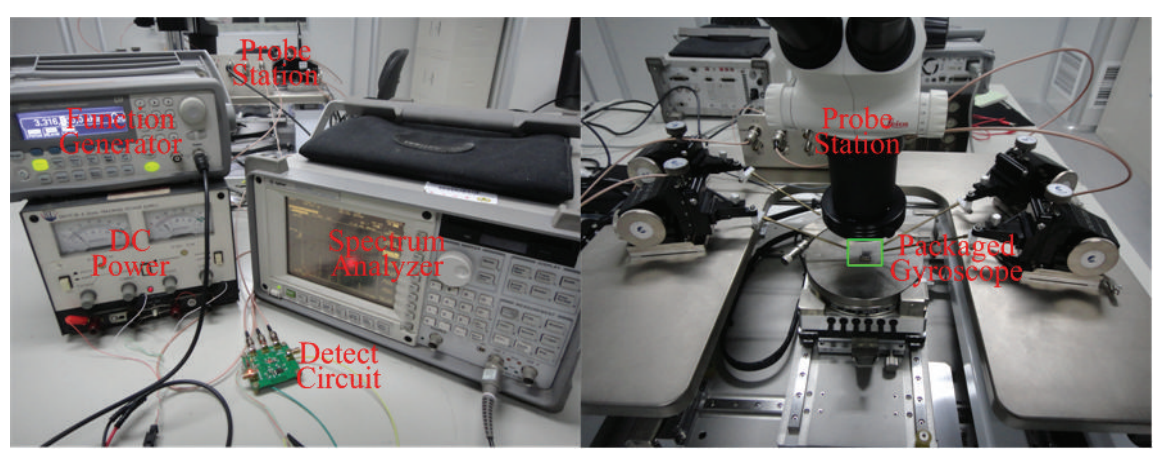

FIGURE 4: Experimental setup for vibration detection.

$$
\begin{aligned}
& =\frac{1}{C_{f}}\left(q_{s 1}-q_{s 2}\right) \\
& =\frac{1}{C_{f}}\left(V_{c} C_{s 1}-V_{c} C_{s 2}\right) \\
& =\frac{V_{c}}{C_{f}} \cdot 2 \Delta C_{s} .
\end{aligned}
$$

Since the squeeze gap in the sense combs is much larger than that in the drive combs, the sense capacitance between the squeeze faces is ignored and the approximate sense capacitance change is proportional to the displacement $x$; that is, $\Delta C_{s}=\left(\partial C_{s} / \partial x\right) x$. Thus, if $x=A \sin \left(2 \pi f_{d} t+\phi\right)$, the output voltage is

$$
\begin{aligned}
V_{o} & =\frac{1}{C_{f}} \cdot \frac{2 \partial C_{s}}{\partial x} \cdot A \sin \left(2 \pi f_{d} t+\phi\right) \cdot V_{a} \sin 2 \pi f_{d} t \\
& =\frac{A V_{a}}{C_{f}} \cdot \frac{\partial C_{s}}{\partial x} \cdot\left(\cos \phi-\cos \left(2 \pi \cdot 2 f_{d} \cdot t\right)\right) .
\end{aligned}
$$

Then, $V_{o}$ is filtered by a high-pass filter and displayed by a spectrum analyzer on which the vibration information of drive mode is at twice the actuation frequency.

The practical experimental setup is illustrated in Figure 4. Through the probe station, a packaged gyroscope sample is connected with the detect circuit. During testing, the actuation frequency of the carrier voltage is consecutively tuned by a step of $0.1 \mathrm{~Hz}$ increasingly or decreasingly between $3310 \mathrm{~Hz}$ and $3330 \mathrm{~Hz}$, and the corresponding amplitude information is recorded by acquiring the value at twice the actuation frequency on the analyzer screen.

3.2. Experimental Results. When $V_{d}=5 \mathrm{~V}, V_{a}=0.5 \mathrm{~V}$, and $f_{d}$ sweeps up slowly from $3310 \mathrm{~Hz}$ to $3330 \mathrm{~Hz}$, the frequency characteristic curve of the drive mode is fitted in red, as shown in Figure 5, using the amplitude data acquired at each frequency step. Similarly, the characteristic curve, while frequency sweeps down, is fitted in black. It is noticed that when the actuation frequency sweeps down, the natural frequency is around $3313 \mathrm{~Hz}$, much lower than that obtained during upwards sweeping, that is, around $3319 \mathrm{~Hz}$. Both frequency response curves bend towards lower frequency.

Experiments are also made on the influence of applied voltage on the nonlinearity of frequency response. In Figure 6, when $V_{d}$ increases from $5 \mathrm{~V}$ to $6 \mathrm{~V}$, the natural frequency of upward-sweeping reduces from $3319 \mathrm{~Hz}$ to $3316 \mathrm{~Hz}$ and that of downward-sweeping decreases from $3313 \mathrm{~Hz}$ to $3308 \mathrm{~Hz}$. Thus, increasing $V_{d}$ makes the spring softening phenomena more evident. Similarly, as seen in Figure 7, increasing $V_{a}$ also aggravates the nonlinear softening characteristic. 


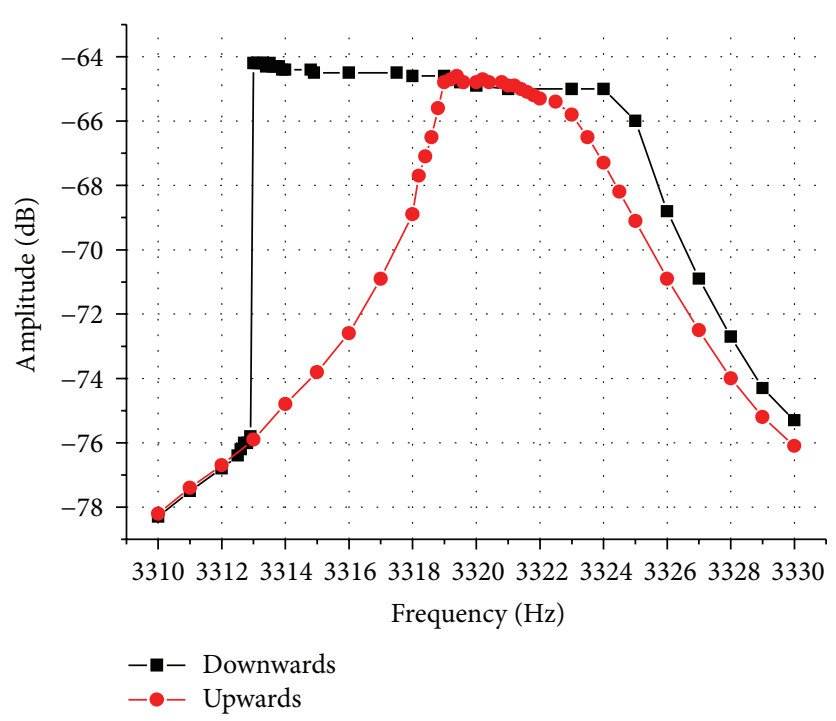

FIGURE 5: Measured amplitude-frequency characteristic of drive mode.

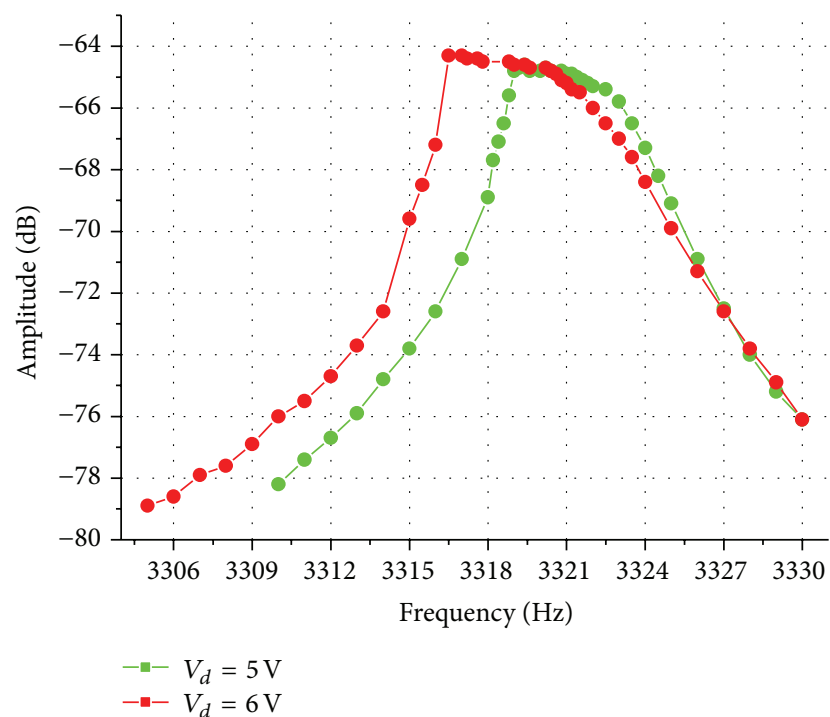

(a)

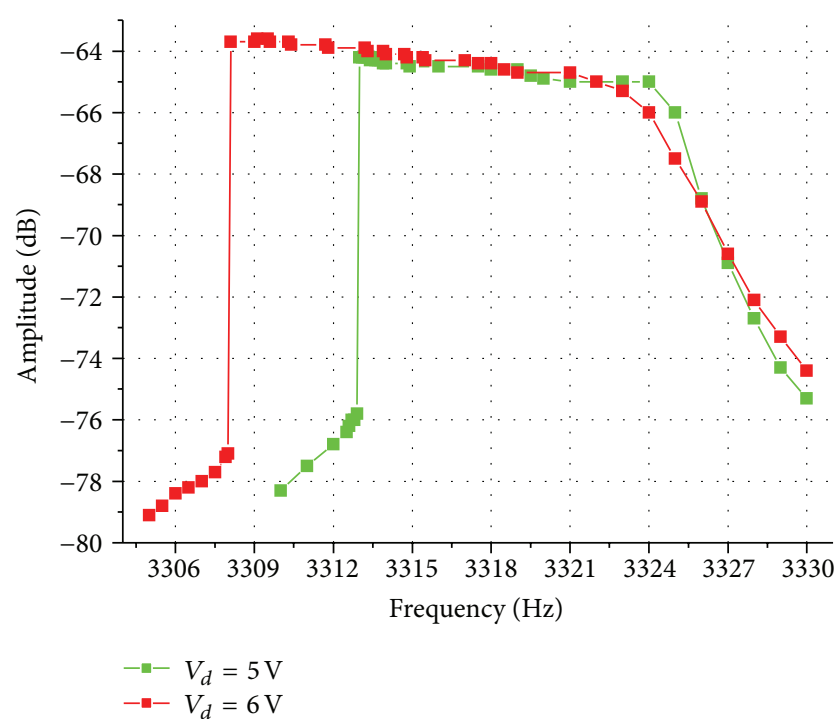

(b)

FIGURE 6: Measured dependence of amplitude-frequency characteristic on DC bias $V_{d}$. (a) Frequency sweeps up. (b) Frequency sweeps down.

\section{Numerical Characterization}

4.1. Simulink Model. Matlab and Simulink are used to determine the motion of the proof mass as well as its frequency response characteristic. Parameters, which are given in Table 1, are firstly defined in the Matlab code and then transferred to the Simulink model for simulation. Figure 8 gives the Simulink model, in which an S-Function module is used to construct (9), differential voltage is applied, and the response is monitored by oscilloscopes. By simulating the model, the stable solution of displacement and velocity is obtained. Besides, the amplitude-frequency characteristic curve is determined by fitting the stable vibration amplitude acquired at each actuation frequency. This model is also used to determine and compare the response under different initial status and voltage loads. Here, the actuation frequency $f_{d}$ is swept in two ways. Within the frequency range of interest, one sweeping method is to increase frequency consecutively by a certain step and the other is to decrease it by the same step. It must be pointed out that, at each frequency sweeping step, the initial status of the displacement and velocity as well as the phase of applied voltage is maintained equal to the value at the finish time of the previous frequency step.

4.2. Influence of Initial Status. When drive voltage is applied with $V_{d}=5 \mathrm{~V}, V_{a}=0.5 \mathrm{~V}$, and $f_{d}=3315 \mathrm{~Hz}$, the phase plane portrait of (9) is illustrated in Figure 9, in which the arrow tells the progressing trend of the nondimensional displacement and velocity along with time [18]. Besides, three 


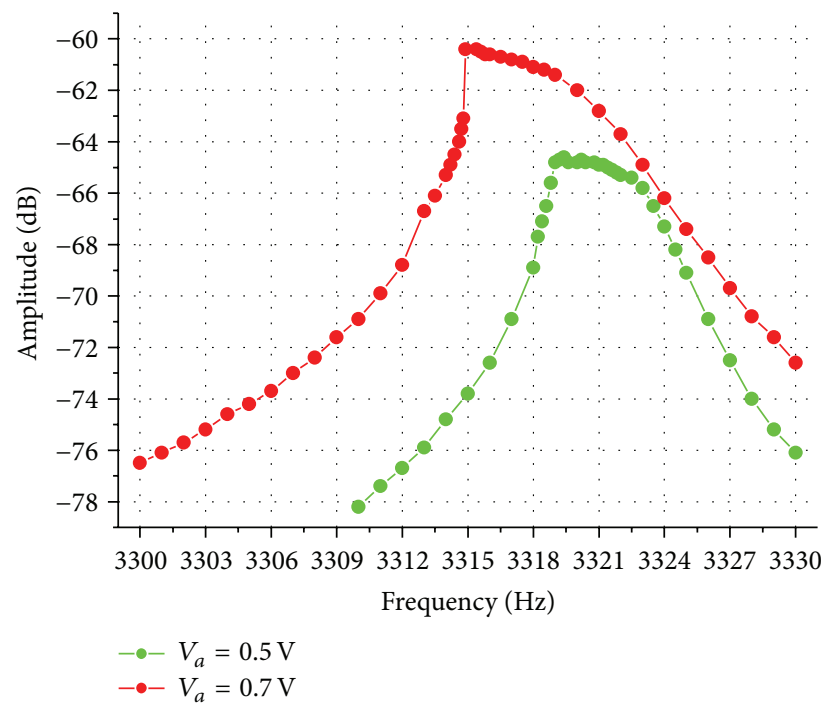

(a)

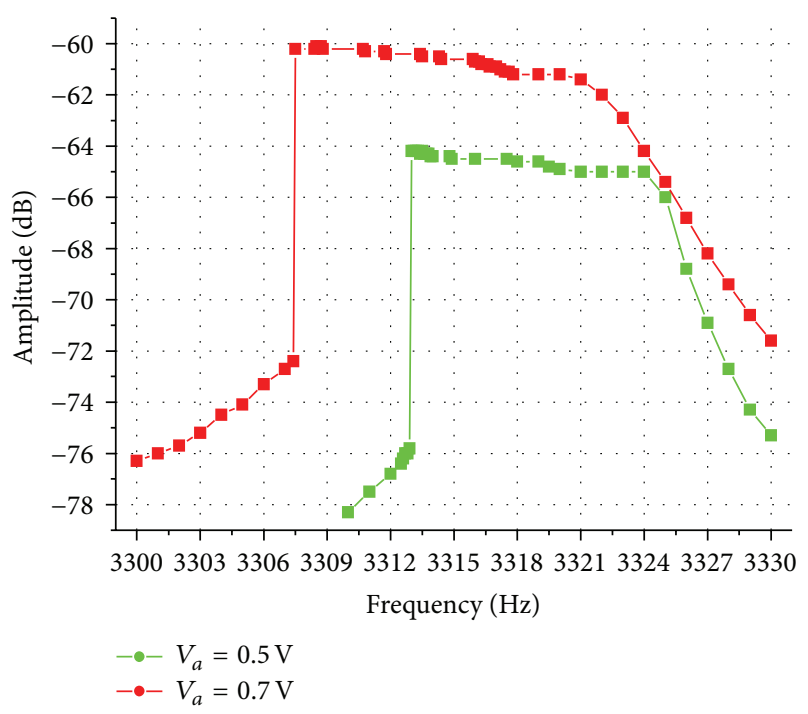

(b)

FiguRE 7: Measured dependence of amplitude-frequency characteristic on amplitude of AC voltage $V_{a}$. (a) Frequency sweeps up. (b) Frequency sweeps down.

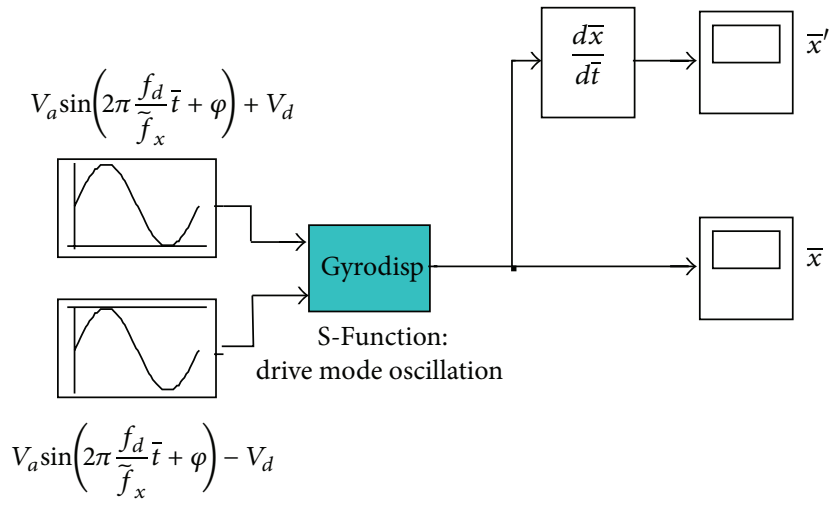

FIGURE 8: Simulink model for solving nonlinear dynamics of drive mode.

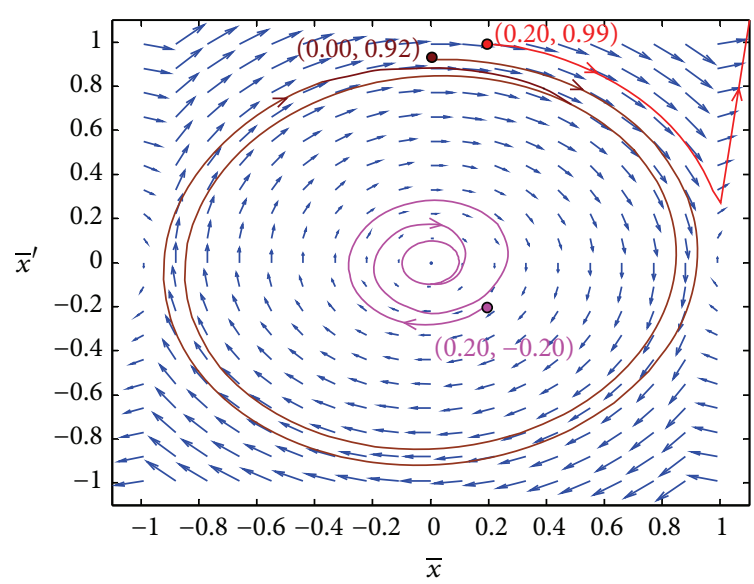

FIgURE 9: Phase plane portrait for drive mode under different initial status. The $x$-axis refers to the nondimensional displacement and the $y$-axis refers to its velocity.

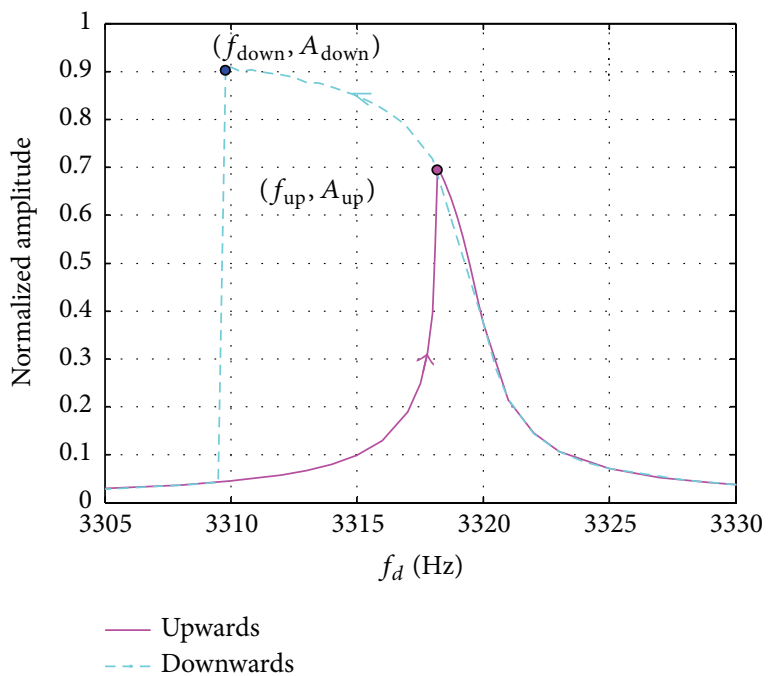

FIGURE 10: Amplitude-frequency characteristic curve of drive mode.

curves in the graph refer to the equivalent transient response trajectories under different initial status. It is observed that the nondimensional stable amplitude of displacement is around 0.1 when initial status is $\left(\bar{x}_{0}, \bar{x}_{0}^{\prime}\right)=(0.20,-0.20)$, while it is around 0.85 when $\left(\bar{x}_{0}, \bar{x}_{0}^{\prime}\right)=(0.00,0.92)$. In addition, the trajectory is divergent when $\left(\bar{x}_{0}, \bar{x}_{0}^{\prime}\right)=(0.20,0.99)$. It is easily concluded that the stable motion of drive mode is dependent on the initial motion status of the proof mass when voltage is applied as here.

4.3. Amplitude-Frequency Response. The Simulink model in Figure 8 and the parameters in Table 1 are reused to obtain the characteristic curve of frequency response while voltage 

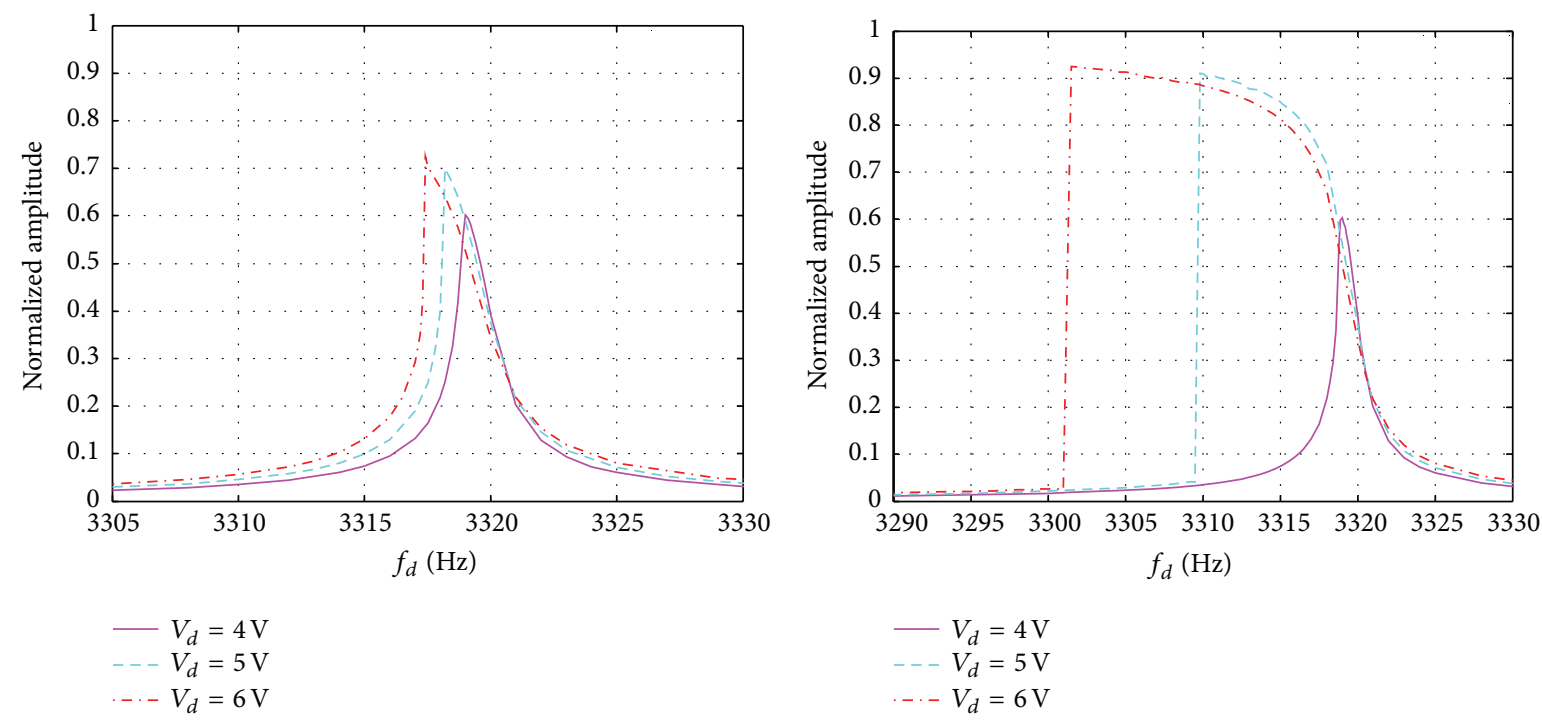

(a)

(b)

FIGURE 11: Dependence of amplitude-frequency characteristic on DC bias $V_{d}$. (a) Frequency sweeps up. (b) Frequency sweeps down.
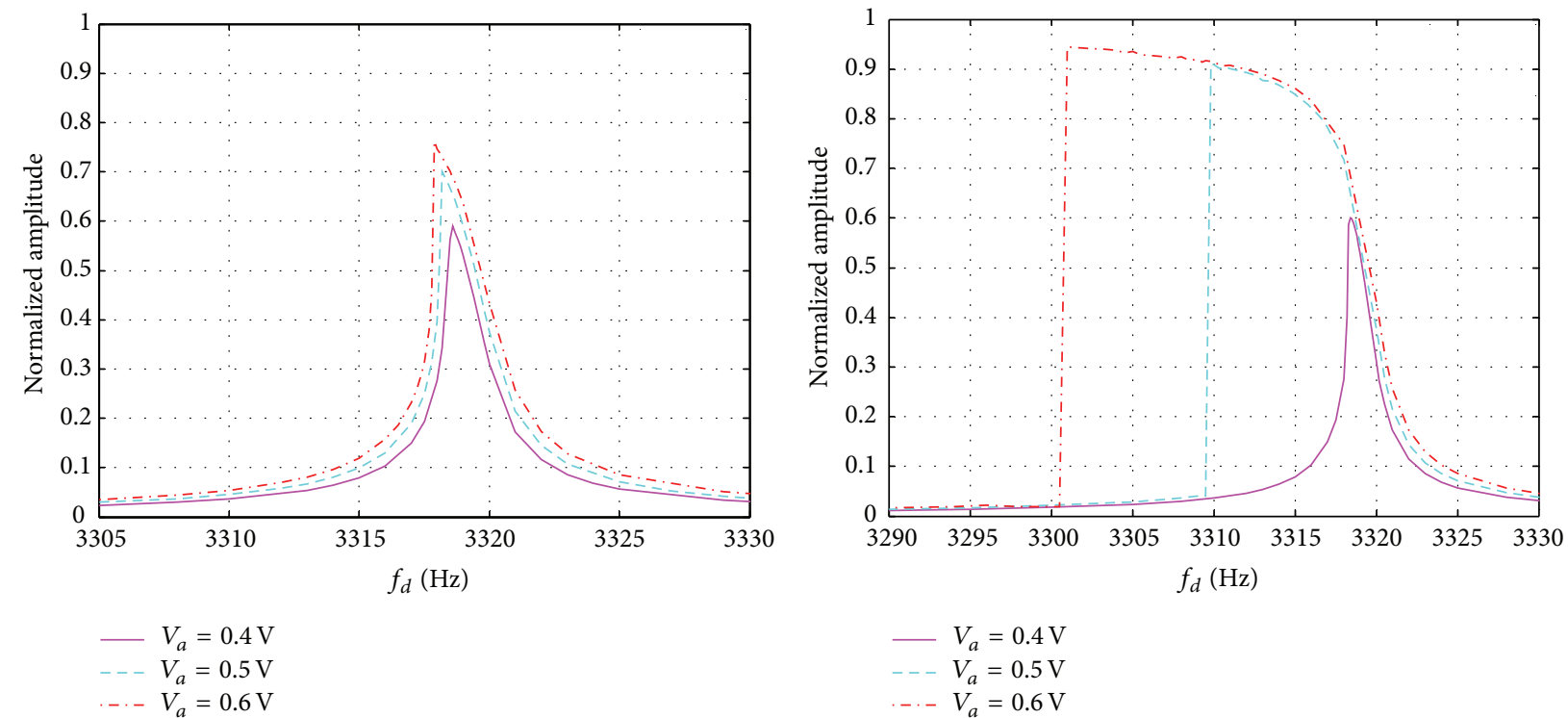

(a)

(b)

FIGURE 12: Dependence of amplitude-frequency characteristic on amplitude of AC voltage $V_{a}$. (a) Frequency sweeps up. (b) Frequency sweeps down.

is applied as $V_{d}=5 \mathrm{~V}, V_{a}=0.5 \mathrm{~V}$, and the actuation frequency $f_{d}$ is increased and decreased consecutively by a step size of $0.5 \mathrm{~Hz}$ between $3305 \mathrm{~Hz}$ and $3330 \mathrm{~Hz}$. Besides, the initial status at the start frequency is $\left(\bar{x}_{0}, \bar{x}_{0}^{\prime}\right)=(0,0)$. The corresponding stable vibration amplitude at each frequency step is collected to fit characteristic curves.

Figure 10 gives the simulated characteristic curves, in which the pink solid line and the blue dashed line refer to the responses while frequency sweeps up and down, respectively. It is noticed that two natural frequencies $f_{\text {up }}$ and $f_{\text {down }}$ appear in frequency response curves. The curves both bend towards lower frequency, manifesting high nonlinearity. During sweeping up, when the actuation frequency gets near $f_{\text {up }}$, the amplitude rushes to the peak $A_{\text {up }}$ and then descends gradually. Similarly, during sweeping down, the amplitude ascends slowly to the peak $A_{\text {down }}$ and then drops dramatically at the frequency $f_{\text {down }}$. It is also seen that two natural frequencies are different with $f_{\text {down }}<$ $f_{\text {up }}<\tilde{f}_{x}$, and their corresponding peak amplitude is not the same with $A_{\text {down }}>A_{\text {up }}$. Besides, when $f_{d}=$ $3315 \mathrm{~Hz}$, the obtained downwards-sweeping amplitude is over 7 times larger than the upwards-sweeping amplitude 


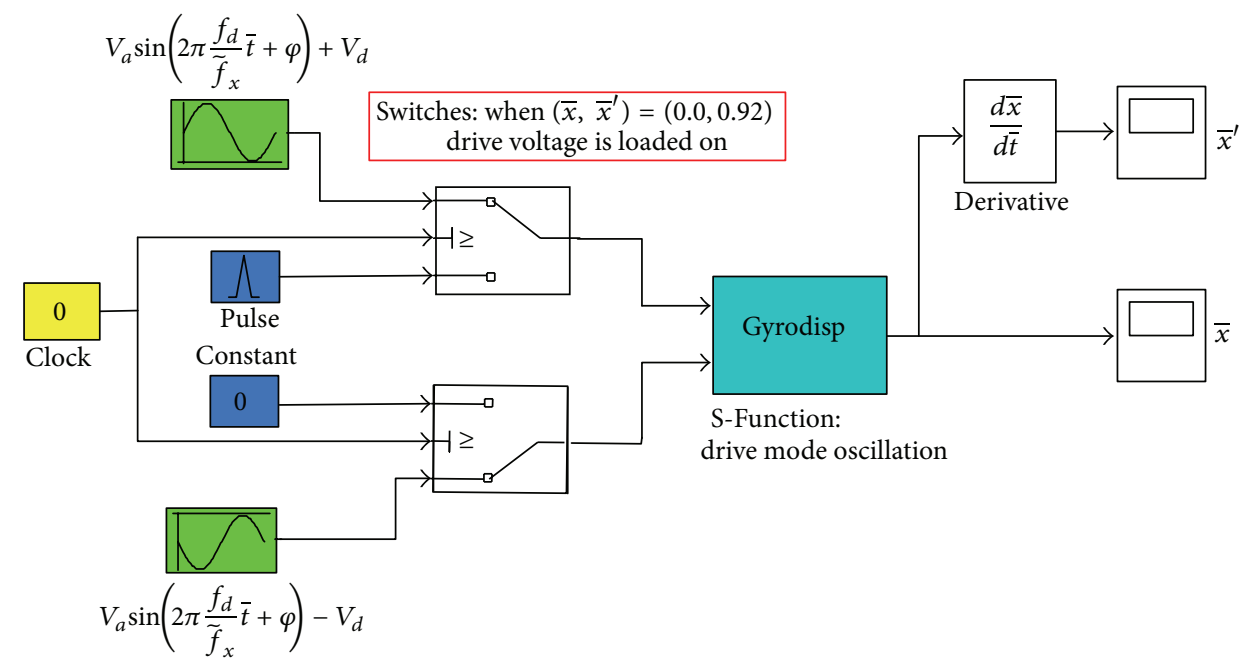

FIGURE 13: Systematic schematic for exciting the nonlinear vibration of drive mode.

TABLE 1: Structural parameters for drive mode of silicon gyroscope.

\begin{tabular}{lcc}
\hline Parameter & Value & Unit \\
\hline Proof mass $m_{x}$ & 1.36 & $\mathrm{mg}$ \\
Linear natural frequency $\tilde{f}_{x}$ & 3320 & $\mathrm{~Hz}$ \\
Quality factor $Q_{x}$ & 2800 & - \\
Comb actuator & & \\
$N$ & 900 & - \\
$h$ & 60 & $\mu \mathrm{m}$ \\
$l_{c 2}$ & 10 & $\mu \mathrm{m}$ \\
$e$ & 4 & $\mu \mathrm{m}$ \\
$d$ & 4 & $\mu \mathrm{m}$ \\
\hline
\end{tabular}

and even larger than $A_{\text {up }}$. In the downwards-sweeping curve, there is a flat region with relatively large amplitude before $f_{\text {down }}$ is reached. Thus, the nonlinear downwards-sweeping amplitude-frequency characteristic curve is very attractive for performance enhancement of silicon gyroscopes.

By using the same Simulink model, some investigation is made on the dependence of the amplitude-frequency characteristic on applied voltage. Simulations are made by changing parameters $V_{d}$ and $V_{a}$ separately. The simulated results are shown in Figures 11 and 12. When $V_{d}$ and $V_{a}$ increase, the two natural frequencies obtained by different sweeping methods both descend but their corresponding peak amplitude ascends. Besides, the decrement of $f_{\text {down }}$ is much larger than that of $f_{\text {up. }}$. It can be concluded that the frequency response nonlinearity can be adjusted by $V_{d}$ and $V_{a}$ and the flat region of the downwards-sweeping curve grows larger when nonlinearity is intensified.

\section{Excitation of Nonlinear Vibration}

According to Figure 9, when the initial status of vibration is $\left(\bar{x}_{0}, \bar{x}_{0}^{\prime}\right)=(0.00,0.92)$ and differential voltages
$0.5 \sin 6.2737 \bar{t}+5$ and $0.5 \sin 6.2737 \bar{t}-5$ are applied on drive combs, the vibration of the drive mode will be stabilized at relatively large amplitude, which is especially attractive for improving the scale factor of silicon gyroscopes. Therefore, Figure 13 illustrates how to excite the nonlinear vibration with larger amplitude. A triangular impulse is firstly loaded to start the motion of drive mode and the vibration displacement and velocity are recorded. As shown in Figure 14, the impulse intensity is adjusted to make the motion status $\left(\bar{x}_{0}, \bar{x}_{0}^{\prime}\right)=$ $(0.00,0.92)$ appear at some time. Thus, the differential drive voltage is loaded at this time. The nonlinear response, as illustrated in Figure 15, is obtained by simulating the model in this way. It is noticed that when $f_{d}$ changes consecutively from $3315 \mathrm{~Hz}$ and $3314 \mathrm{~Hz}$ to $3316 \mathrm{~Hz}$, the stable displacement amplitude varies from 0.848 and 0.867 to 0.823 and the phase relative to the normalized sine voltage is maintained around -108.6 degrees.

According to the numerical and experimental characteristics, the nonlinear vibration with larger amplitude can be actuated by decreasing the drive frequency consecutively with a certain frequency step and time interval. Thus, the nonlinear vibration in the downward sweeping curve can be excited by monitoring or gradually accumulating the status of vibration. Finally, the novel drive methodology makes drive mode vibrate at large amplitude and it is robust with no drops of amplitude and phase when the drive frequency fluctuates.

\section{Discussion}

When differential voltages $V_{a} \sin 2 \pi f_{d} t+V_{d}$ and $V_{a} \sin 2 \pi f_{d} t-$ $V_{d}$ are applied on drive stators with $V_{d}=5 \mathrm{~V}$ and $V_{a}=$ $0.5 \mathrm{~V}$, the amplitude-frequency characteristic curves obtained by experiments and simulations are in good agreement. It is displayed that the frequency characteristic curves bend towards lower frequency with the natural frequency much smaller than the initial value. Besides, the peak vibration 


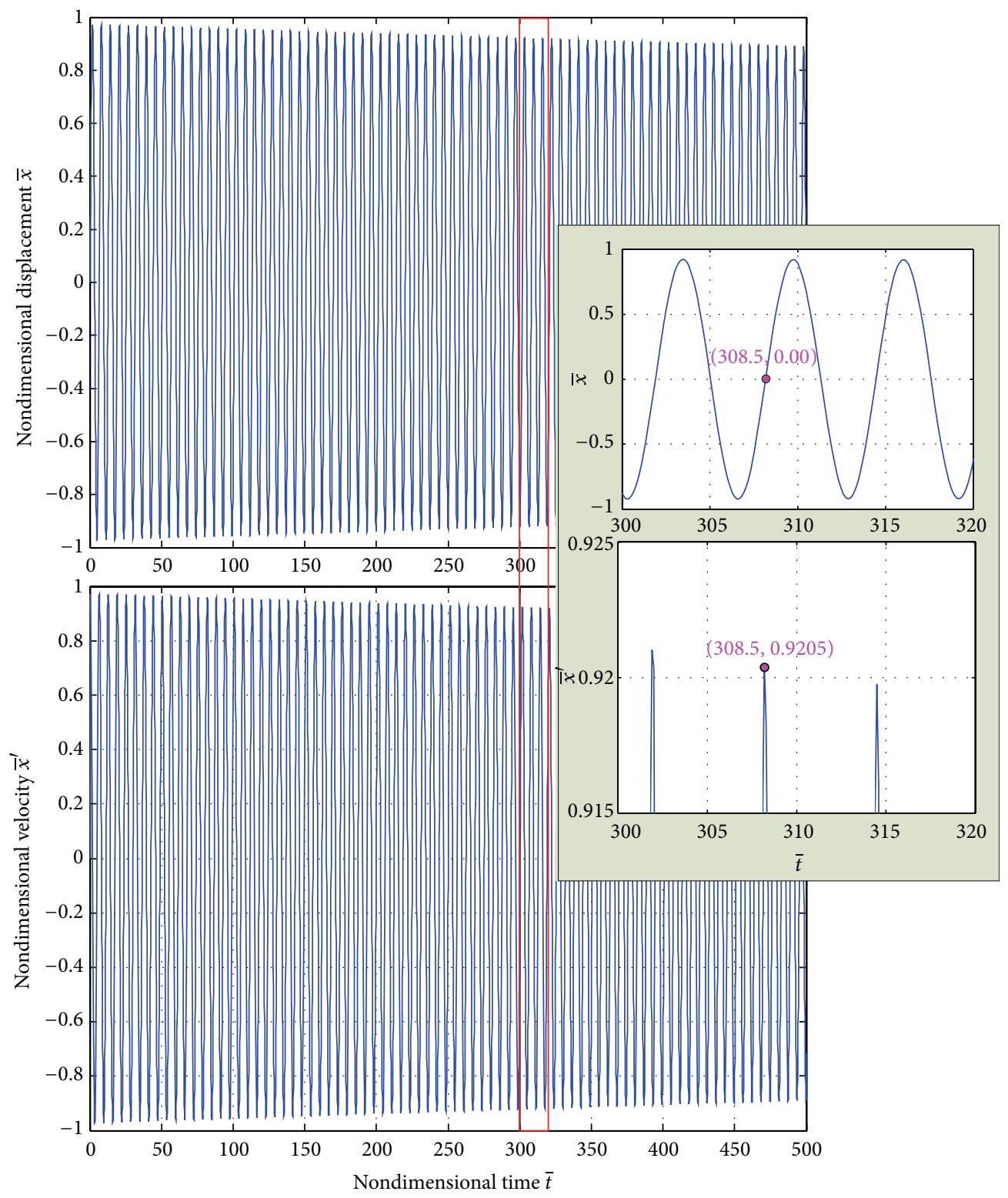

FIGURE 14: Response of drive mode when a single triangular pulse strike is loaded.

amplitude and the natural frequency, obtained by different sweeping methods, are not the same.

According to (8), it is known that $K_{\text {elinear }}$ and $K_{2 \text { enon-linear }}$ lead to reduction of the natural frequency $\omega_{x}$ while $K_{\text {non-linear }}$ raises it. In addition, $K_{2 e n o n-l i n e a r}$ makes the frequency response curves bend towards lower frequency while $K_{\text {non-linear }}$ makes them bend towards higher frequency. Thus, the spring softening phenomena in Figures 5 and 10 imply the relationship between the nonlinear stiffness coefficients in (8); that is, $K_{2 e n o n-l i n e a r}>K_{\text {non-linear }}$.

Based on the definition of $K_{\text {elinear }}$ and $K_{2 e n o n-l i n e a r}$, increasing $V_{d}$ or decreasing $l_{c 2}$ makes electroelastic stiffness larger, leading to more evident spring softening phenomena. Besides, according to (8), the nonlinear stiffness is also increased by displacement enlargement. Thus, increasing $V_{d}$ and $V_{a}$, which makes the vibration amplitude of the proof mass larger, aggravates spring softening phenomena. The same influence of applied voltage on the amplitude-frequency characteristic is indicated by the experimental results in Figures 6 and 7 as well as the numerical analysis in Figures 11 and 12.

In the nonlinear amplitude-frequency characteristic curves, though the vibration amplitude and the natural frequency are multivalued, the flat region of downwardssweeping curves with extremely large amplitude is useful for improving gyroscope's performance, such as sensitivity and robustness.

It is numerically proved that the vibration of the drive mode ends with different stable amplitude under different initial motion status, when applied voltage is large enough. 


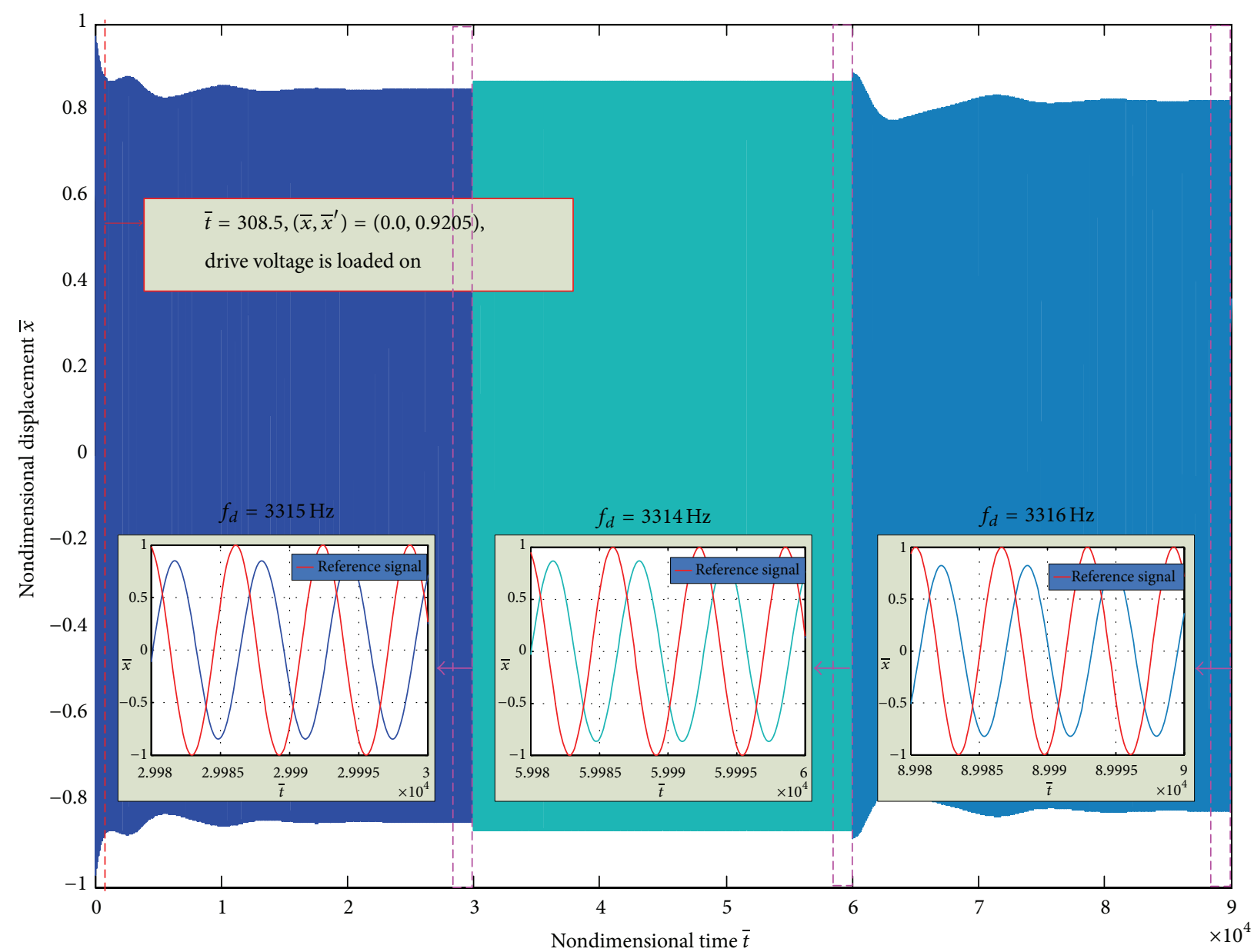

FIGURE 15: Nonlinear vibration of drive mode when differential drive voltage is loaded.

Thus, the nonlinear vibration with larger amplitude can be excited by loading drive voltage at the specific status or decreasing the drive frequency gradually by a certain frequency and time step. The obtained amplitude in the downward sweeping curve is much larger than the amplitude in the linear frequency response curve. Besides, the amplitude and the phase of vibration are less influenced by the fluctuation of the actuation frequency. It can be concluded that this novel drive methodology is beneficial for performance improvement of silicon vibratory gyroscopes.

\section{Conclusion}

In this paper, the nonlinear frequency softening characteristic of the drive mode in the $Z$-axis silicon microgyroscope designed by our research team is determined by analytical, experimental, and numerical methods, results of which show good agreement qualitatively. It is illustrated that the spring softening phenomena of the amplitude-frequency characteristic appear when applied voltage and the vibration amplitude are large enough. Besides, it is also proved that applied voltage plays a significant role in the nonlinear vibration of the gyroscope, so the nonlinearity can be tuned by drive voltage.
Moreover, under nonlinear vibration, the wide flat region with large amplitude in the downwards-sweeping amplitudefrequency characteristic curve is attractive for improving gyroscope's performance. The nonlinear vibration of large amplitude can be excited by applying drive voltage at a certain motion status or gradually decreasing the actuation frequency. Besides, the large amplitude and the phase of vibration are maintained when the actuation frequency fluctuates. Thus, for silicon microgyroscopes, it can be a promising drive method to make the drive mode vibrate at the large amplitude in the flat region of the nonlinear amplitudefrequency characteristic curve.

\section{Conflict of Interests}

The authors declare that there is no conflict of interests regarding the publication of this paper.

\section{Acknowledgment}

This work is supported by preresearch fund Project of 9140A09011313JW06119. 


\section{References}

[1] S. Wang, L. Huang, and B. Yang, Weiguanxing yibiao yu weixitong, Weapon Industry Press, Beijing, China, 2011.

[2] N. Yazdi, F. Ayazi, and K. Najafi, "Micromachined inertial sensors," Proceedings of the IEEE, vol. 86, no. 8, pp. 1640-1658, 1998.

[3] A. M. Shkel, C. Acar, and C. Painter, "Two types of micromachined vibratory gyroscopes," in Proceedings of the 4th IEEE Conference on Sensors, pp. 531-536, Irvine, Calif, USA, November 2005.

[4] A. M. Shkel, "Micromachined gyroscopes: challenges, design solutions, and opportunities," in Smart Structures and Materials: Smart Electronics and MEMS, vol. 4334 of Proceedings of SPIE, pp. 74-85, Newport Beach, Calif, USA, August 2001.

[5] W. A. Clark, Micromachined vibratory rate gyroscopes [Ph.D. thesis in Engineering-Electrical Engineering and Computer Science], University of California, Berkeley, Calif, USA, 1997.

[6] W. Geiger, J. Bartholomeyczik, U. Breng et al., "MEMS IMU for AHRS applications," in Proceedings of the IEEE/ION Position, Location and Navigation Symposium (PLANS '08), pp. 225-231, May 2008.

[7] A. Sharma, F. M. Zaman, B. V. Amini, and F. Ayazi, "A high$\mathrm{Q}$ in-plane SOI tuning fork gyroscope," Proceedings of IEEE Sensors, vol. 1, pp. 467-470, 2004.

[8] A. Sharma, M. F. Zaman, and F. Ayazi, "A sub- $0.2^{\circ} / \mathrm{hr}$ bias drift micromechanical silicon gyroscope with automatic CMOS mode-matching," IEEE Journal of Solid-State Circuits, vol. 44, no. 5, pp. 1593-1608, 2009.

[9] F. Braghin, F. Resta, E. Leo, and G. Spinola, "Nonlinear dynamics of vibrating MEMS," Sensors and Actuators, A: Physical, vol. 134, no. 1, pp. 98-108, 2007.

[10] A. M. Elshurafa, K. Khirallah, H. H. Tawfik, A. Emira, A. K. S. Abdel Aziz, and S. M. Sedky, "Nonlinear dynamics of spring softening and hardening in folded-mems comb drive resonators," Journal of Microelectromechanical Systems, vol. 20, no. 4, Article ID 5772897, pp. 943-958, 2011.

[11] R. M. C. Mestrom, R. H. B. Fey, K. L. Phan, and H. Nijmeijer, "Simulations and experiments of hardening and softening resonances in a clamped-clamped beam MEMS resonator," Sensors and Actuators, A: Physical, vol. 162, no. 2, pp. 225-234, 2010.

[12] R. M. C. Mestrom, R. H. B. Fey, J. T. M. van Beek, K. L. Phan, and H. Nijmeijer, "Modelling the dynamics of a MEMS resonator: simulations and experiments," Sensors and Actuators A: Physical, vol. 142, no. 1, pp. 306-315, 2008.

[13] M. Palaniapan and L. Khine, "Nonlinear behavior of SOI freefree micromechanical beam resonator," Sensors and Actuators, A: Physical, vol. 142, no. 1, pp. 203-210, 2008.

[14] T. Veijola and T. Mattila, "Modeling of nonlinear micromechanical resonators and their simulation with the harmonic-balance method," International Journal of RF and Microwave ComputerAided Engineering, vol. 11, no. 5, pp. 310-321, 2001.

[15] B. Yang, B. Zhou, S. Wang, L. Huang, and Y. Yin, "A quadrature error and offset error suppression circuitry for Silicon MicroGyroscope," in Proceedings of the 3rd IEEE International Conference on Nano/Micro Engineered and Molecular Systems (NEMS '08), pp. 422-426, January 2008.

[16] Y. Yin, S. Wang, C. Wang, and P. Sheng, "Structural scheme design and simulation of structure-decoupled dual-mass MEMS gyroscope," Journal of Southeast University, vol. 38, no. 5, pp. 918-922, 2008.
[17] Y. Ni, H. Li, L. Huang, and L. Zhao, "Design and test of quadrature correction structure for silicon micro-gyroscope," Journal of Southeast University: Natural Science Edition, vol. 43, pp. 1227-1231, 2013.

[18] W. Zhang, G. Meng, and D. Chen, "Stability, nonlinearity and reliability of electrostatically actuated MEMS devices," Sensors, vol. 7, no. 5, pp. 760-796, 2007. 

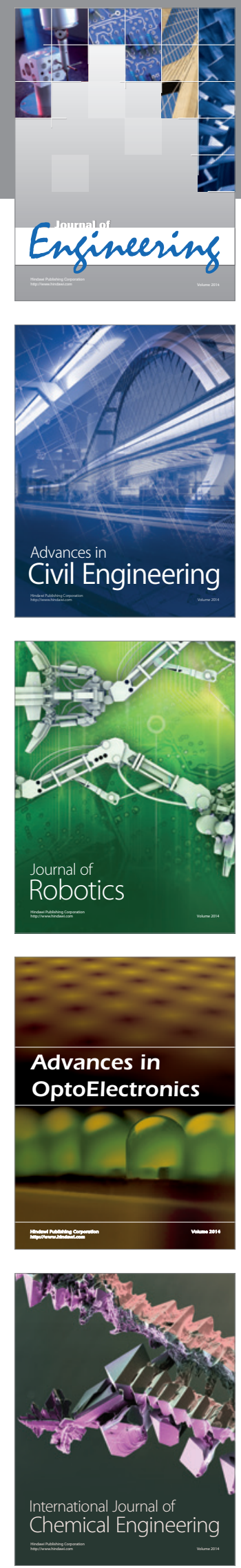

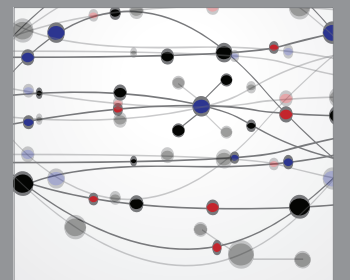

The Scientific World Journal
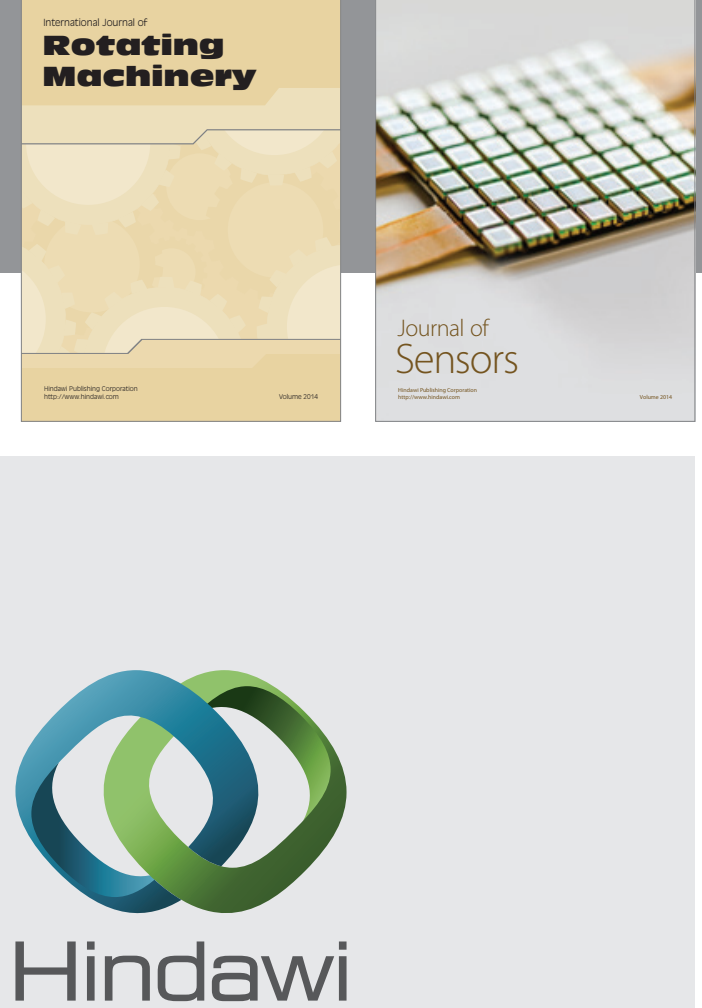

Submit your manuscripts at http://www.hindawi.com
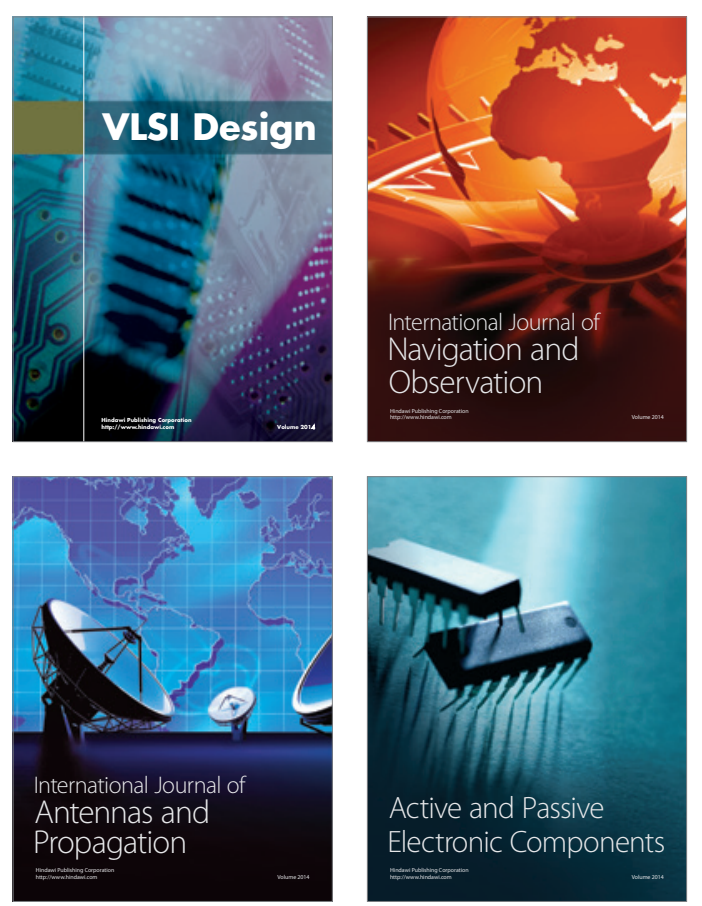
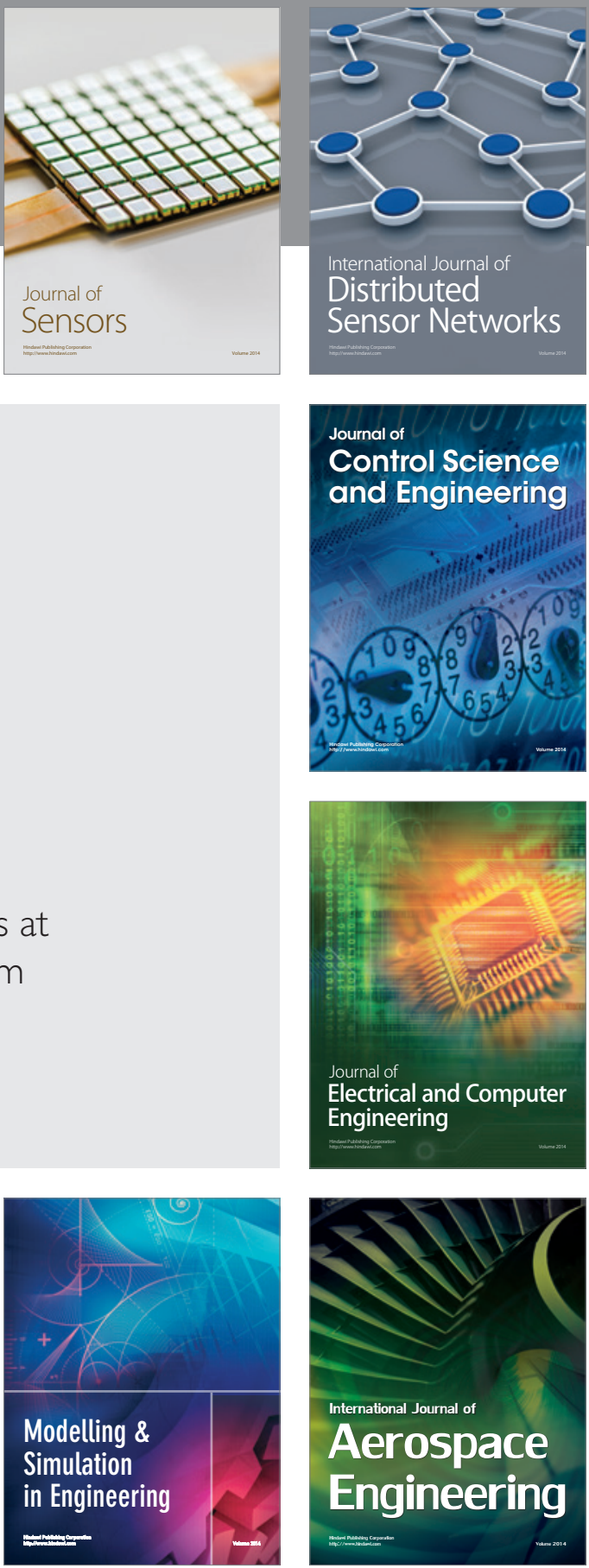

Journal of

Control Science

and Engineering
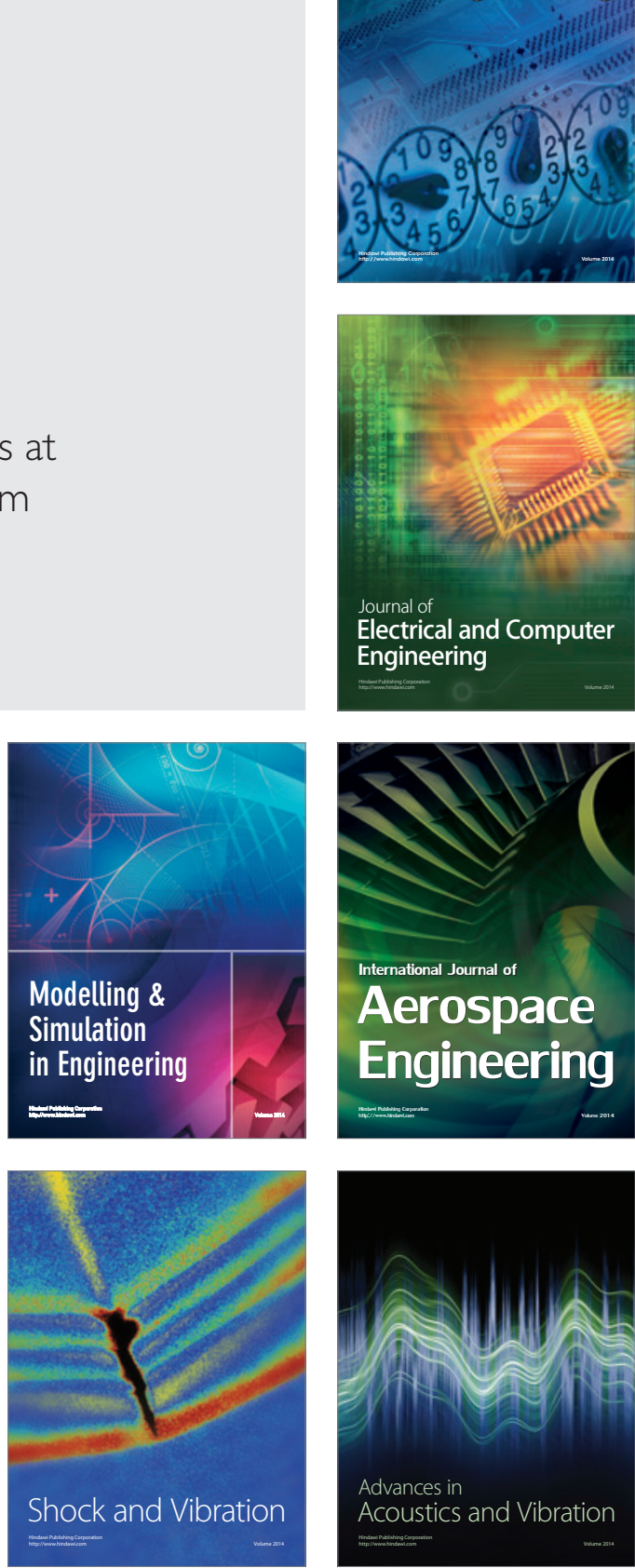